\title{
Mineralized Tissue Changes Within the Anterior Cruciate Ligament Entheses Following a Tear
}

\author{
Nicholas Nguyen ${ }^{1}$, Stephen Schlecht ${ }^{12}$ \\ ${ }^{1}$ Indiana University School of Medicine; ${ }^{2}$ Department of Orthopaedic Surgery
}

Background: Thirty-percent of patients under 20 years of age suffer a primary ACL graft failure. Young patients show significant bone loss at the femoral ACL-bone insertion site (enthesis). We hypothesize that active bone resorption during ACL graft fixation contributes to poor graft survival in the young. We investigated distal femoral and entheseal bone morphology changes after injury in a novel mouse model to determine if previously reported post-injury patient condition anti-translates to the mouse. Confirmation of a similar condition across species will allow us to use this model to spatially and temporally track physiologic processes within this critical region.

Methods: We induced an in vivo ACL rupture in 24 10-week C57BL/6J female mice and sacrificed them at 7 and 28 post-injury. Both loaded ipsilateral and non-loaded contralateral knees were 3D imaged and distal femoral and ACL entheseal cortical regions have begun being analyzed. Traits analyzed include 1) cortical (Ct.) mean gray-value density, area (Ar.), thickness (Th.), and bone volume fraction (BV/TV) Statistics: two-way ANOVA and Tukey posthoc.

Results: So far, mice are temporally demonstrating bone differences between injured and noninjured knees similar to that of patients at the time of ACL reconstructive surgery. By 7 days there is a steep decline in whole distal femoral epiphysis and ACL femoral entheseal Ct.Ar ($19.84 \%$ and $-11.46 \%$, respectively) and Ct.Th $(-8.09 \%$ and $-3.52 \%$, respectively). The trend in Ct.Ar loss holds at 28 days $(-40.95 \%$ and $-8.32 \%$, respectively), but not in Ct.Th, which substantially increases $(6.26 \%$ and $11.05 \%$, respectively).

Conclusion: It appears bone loss is rapid following injury, and that by 28 days there is increased porosity within the cortex with new periosteal/endosteal bone formation counteracting this. If true, particularly within the entheseal region, this may prove problematic for long-term graft outcomes when surgery is performed at the time of significant macrophagic activity. 